\title{
A new algorithm for time-frequency spread coders using multirate filters
}

\author{
K. P. Chan* , L. Chen** and T. Q. Nguyen***
}

*Dept. of Computer Science, University of Hong Kong, Pokfulam Road, Hong Kong

**Dept. of EE, Shantou University, Shantou, Guangdong, P.R. China lchen@mailservs.stu.edu.cn

***Department of Electrical \& Computer Engr., Boston University, 44 Cummington St., Boston, MA 01803

\section{ABSTRACT}

In recent years, filter banks have been shown to be efficient in several emerging signal communication applications. A new class of time-frequency spread coders for transmultiplexer systems using multirate filter banks is presented in this paper. As compared with conventional filter banks designed with stopband attenuation and passband flatness criteria, the user coders in the new algorithms are designed with time and frequency spread criteria. In new the algorithms, the filters are achieved through a cascade of lattice structures and delay chains. Along with the timefrequency property and the reconstruction property, the intercode/intracode correlation property are included in the design algorithms to be optimized. The designed coders are used in the application of digital watermarking of images and demonstrate good performances in JPEG encoding at different qualities.

\section{INTRODUCTION}

In recent years, subband filter banks have received considerable interests among researchers in signal processing and communication applications[1][2] Several transmultiplexer systems in communications applications can be discussed as the synthesis and analysis structures of filter banks, including code division multiple access (CDMA), frequency division multiple access (FDMA), and time division multiple access (TDMA) communication schemes. In this paper, generalized design algorithms are examined for paraunitary subband filter banks in spread spectrum orthogonal transmultiplexers. In the algorithms, the filter lengths are gradually reduced through a cascade of lattice structures and delay chains. The generalized algorithms are modified to improve the spread-response properties of the systems in time and frequency domain. Design algorithms are presented for filter banks (user codes) with minimized auto- and crosscorrelation properties. The designed user coders are used in the application of digital watermarking of images and show good performance in JPEG encoding and other signal processing systems.

\section{FILTER BANKS}

A. Analysis/synthesis systems

Fig. 1 shows the process of signal decomposition and reconstruction in a filter bank. In the analysis system, an input sequence $x(n)$ is decomposed into a set of subband sequences via the analysis filters $H_{i}(n)$ and the decimators. In the synthesis system, the subband sequences $y_{i}(n)$ are upsampled by the interpolators and recombined by the synthesis filters $F_{i}(n)$ to reconstruct the original signal. The decimators following the analysis filters and the interpolators preceding the synthesis filters are depicted as blocks with downsampling arrows $(\downarrow M)$ and upsampling arrows $(\uparrow M)$, respectively.

\section{B. Parauntary filter banks}

An important subset of filter banks is the subband system in which the polyphase matrix $E(z)$ satisfies the paraunitary property [1]

$$
\widetilde{E}(z) E(z)=I_{M}, \text { for all } z
$$

where $\widetilde{E}(z)$ is the transpose-conjugate of $\boldsymbol{E}(z)$, i.e., $\widetilde{E}(z)=E^{T}\left(z^{-1}\right)$. The paraunitary systems in (1) can be treated as an extension of the memoryless orthogonal block transforms to linear, linear-timeinvariant systems with memory. Filter banks with paraunitary property have several additional advantages. For examples, the synthesis filters $F_{i}(z)$, $0 \leq i \leq M-I$ have the same length as the analysis filters $\boldsymbol{H}_{i}(z), 0 \leq i \leq M-l$ and can be obtained by time-reversal of the analysis filter coefficients without matrix inversion. Paraunitary systems can be traced back to classic network synthesis systems. An interesting characteristics of paraunitary filter banks is that they can be regarded as an extension of nonoverlapping block transforms to overlapping block transforms[2].

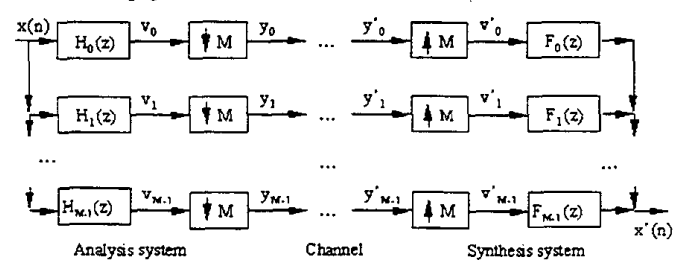

Fig. I Analysis/synthesis systems in Filter banks

\section{SPREAD SPECTRUM}

Transmultiplexer structures

The transmultiplexer model uses a structure displayed in Fig. 2, which is similar to the orthogonal analysis/synthesis filter bank in Fig. 1. In a FDMA type of transmultiplexer, the synthesis and analysis filters $F_{i}(n)$ and $H_{i}(n)$, respectively, are frequency selective and brick-wall shaped. In contrast to FDMA, a TDMA scheme allocates a specific time for each user where the synthesis and analysis

filters $F_{i}(n)$ and $H_{i}(n)$ are delay chains and spectrally allpass. The CDMA approach uses users codes (filters) that are simultaneously spread in both the time and frequency domain.

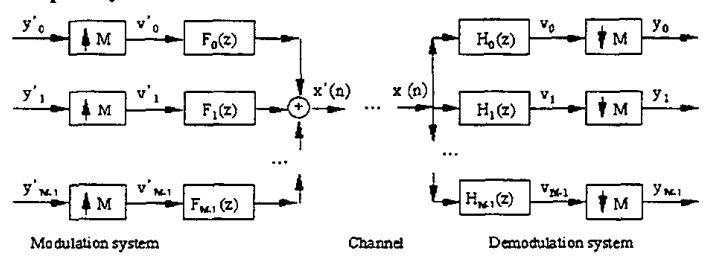

Fig. 2 Synthesis/analysis systems in CDMA systems 


\section{B. Spread-spectrum system}

The techniques of spread spectrum are used in conjunction with modulation schemes and affect the spectrum of the modulated carrier. Spreading signal spectrum reduces the spectral power density (the power transmitted in unit band-width) and desensitizes the communications link to fading or interference[2]. Spread spectrum techniques can be used to enable several communication links to share the same piece of spectrum range and same piece of time interval simultaneously provided that they use uncorrelated frequency hopping or spreading functions.

\section{TIME-FREQUENCY CRITERIA}

Spreading the transmultiplexer codes in both frequency and time-domains as wide as possible is a special property of spread-spectrum coders in CDMA communications. In contrasts with the conventional subband QMF filter banks, which approximate the ideal brick-wall frequency responses[1][2]. The frequency selectivity of conventional subband PR-QMF's (FDM) become orthogonal spread spectrum codes of the desired CDMA type when the time-frequency spread property is properly considered. In addition to the timefrequency spread property, coder correlation properties are considered in the new algorithm and included in the objective function to be optimized in the coder design.

\section{A. Time-spread and frequency-spread}

The time-spread of a subband filter response $\left\{h_{i}(n)\right\}$, $0 \leq i \leq M-I$ characterizes the time location property and is defined by [3]

$$
\sigma_{n_{i}}^{2}=\frac{1}{E_{i}} \sum_{n}\left(n-\bar{n}_{i}\right)^{2}\left|h_{i}(n)\right|^{2},
$$

where the energy $E_{i}$ and time center $\bar{n}_{i}$ of the function $\left\{h_{i}(n)\right\}$ are

$$
E_{i}=\sum_{n}\left|h_{i}(n)\right|^{2}, \bar{n}_{i}=\frac{I}{E_{i}} \sum_{n} n\left|h_{i}(n)\right|^{2} .
$$

The frequency-spread of a subband filter response $\left\{h_{i}(n)\right\}$ represents the localization measure given by

$$
\sigma_{\omega_{i}}^{2}=\frac{1}{2 \pi E_{i}} \int_{-\pi}^{\pi}\left(\omega-\bar{\omega}_{i}\right)^{2}\left|H_{i}\left(e^{j \omega}\right)\right|^{2} d \omega,
$$

where

$$
\begin{gathered}
H_{i}\left(e^{j \omega}\right)=\sum_{n} h_{i}(n) e^{-j \omega m} \\
\bar{\omega}_{i}=\frac{1}{2 \pi E_{i}} \int_{-\pi}^{\pi} \omega\left|H_{i}\left(e^{j \omega}\right)\right|^{2} d \omega
\end{gathered}
$$

B. Intracoder and intercoder correlations The intracode and intercode correlations are important factors in the applications of asynchronous communications systems and in multiple digial watermarking of images. The intracode $R_{i i}(k)$ and intracode correlation $R_{i j}(k)$ are defined as

$$
\begin{aligned}
& R_{i j}(k)=\sum_{n} h_{i}(n) h_{i}(n+k),(k>0, k \in Z) \\
& R_{i j}(k)=\sum_{n}^{n} h_{i}(n) h_{j}(n+k), i \neq j,(\forall k, k \in Z)
\end{aligned}
$$

\section{Objective function}

The lattice structures used in this paper consist of unitary matrices and delay chains proposed in $[4][5][6]$. With the paraunitary reconstruction constraint in (1) satisfied, the subband filter banks optimizes the following objective function

$$
\begin{aligned}
\{J\}_{\max }= & \sum_{i=0}^{M-1}\left(\alpha \sigma_{n_{i}}^{2}+\beta \sigma_{\omega_{i}}^{2}\right) \\
& -\sum_{i=0}^{M-1} \sum_{j=0}^{i}\left(\gamma_{i j} \sum_{k}\left|R_{i j}(k)\right|\right)
\end{aligned}
$$

\section{EXPERIMENTAL RESULTS}

The frequency spectra and impulse responses of a fiveuser spread spectrum CDMA coder are shown in Fig. 3 and Fig.4, respectively. The curves in solid line, dotted line and dashed line represent the first, third and fifth filter, respectively. The second filter and fourth filter are shown with '+' and 'o', respectively. The frequency responses in Fig. 3(a) are spread over the frequency domain and are mixed one another. The corresponding time-domain responses are concentrated and localized, as shown in Fig.4(a) where the first of the five usercodes corresponding the solid line in Fig. 3(a) is displayed. The frequency responses in Fig. 3(b) are concentrated and localized on specific frequency ranges (In the ideal case, the overlaps between the frequency responses vanish). The corresponding time-domain responses are spread over the time domain, as shown in Fig.4(b) where the first of the five user-codes is displayed. Two five-user coders that are spread in both time-domain and frequency-domain are shown Fig. 3(c)(d) and Fig.4(c)(d). The designed coders in Fig.4(a) are employed in the application of digital watermarking of images[7]. Fig. 5(a) is the original image and Fig. 5(b) is the image with watermarking. The watermark was a Gaussian noise of 2500 taps with zero mean and unit variance and was put in the first of the 25 subbands. It was added to the image with a weight of 15 . The peak signal to noise ratio is $27.97 \mathrm{~dB}$ and the watermarking is invisible. The correlation output is 48.21 when the original watermark matches detected watermark and is under 5.12 when the original watermark and the detected watermark mismatch. The JPEG compression was applied to the watermarked image in Photoshop using the "Medium quality" setting. After compression, the watermark can also be retrieved with a correlation output of 29.31. An advantage of the watermarking approach as compared with block transform based schemes is that the filter responses (time-frequency spread coders) are only known by each individual users. 


\section{CONCLUSION}

In this paper, a new class of orthogonal filter banks (spread coders) has been proposed for time-frequency transmultiplexer systems. The user coders in the new algorithms were designed with time and frequency spread property and the intercode/ intracode correlation property. The designed coders are used in the application of digital watermarking of images.

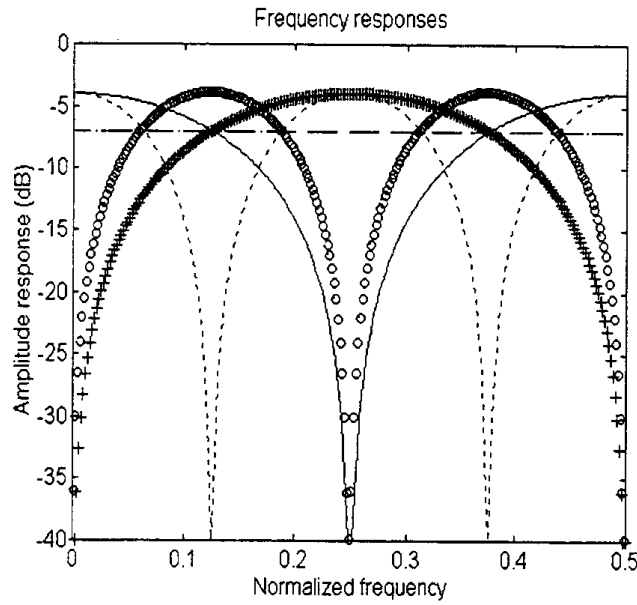

(a)

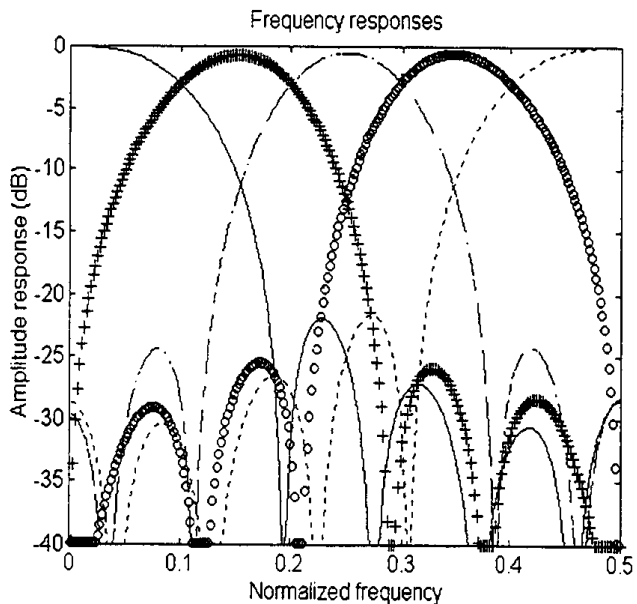

(b)

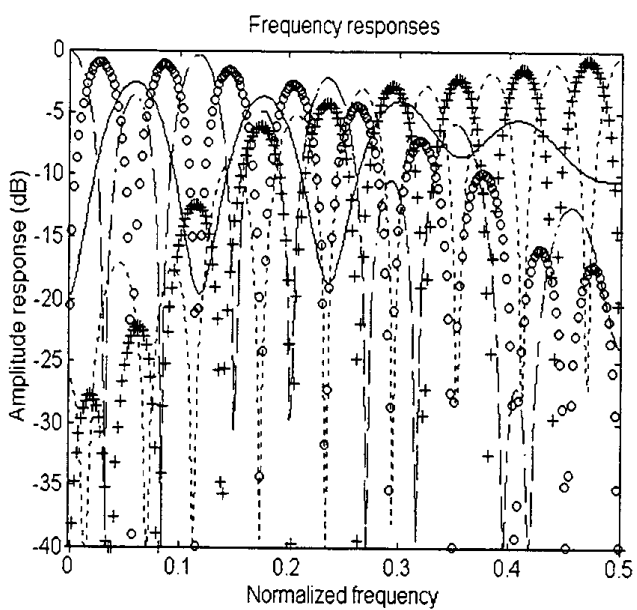

(c)

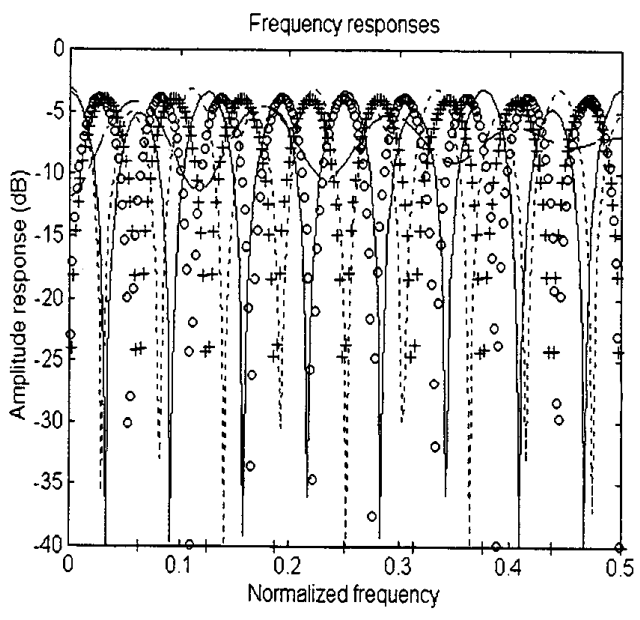

(d)

Fig. 3 Frequency-domain responses of a five-user spread spectrum CDMA coders

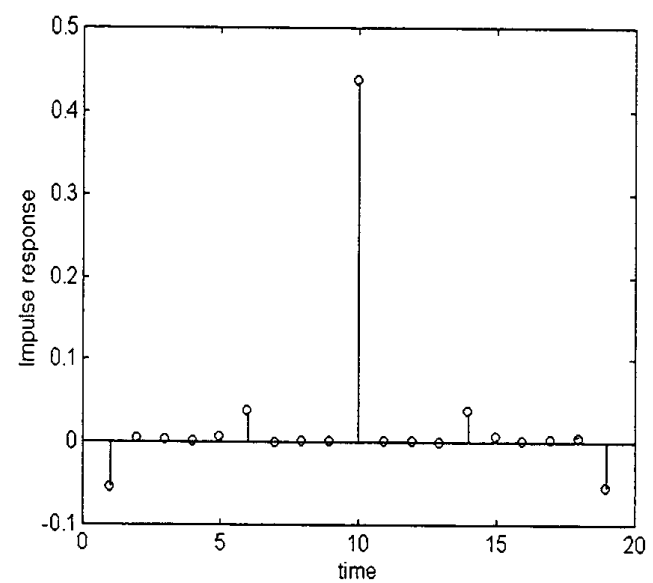

(a) 


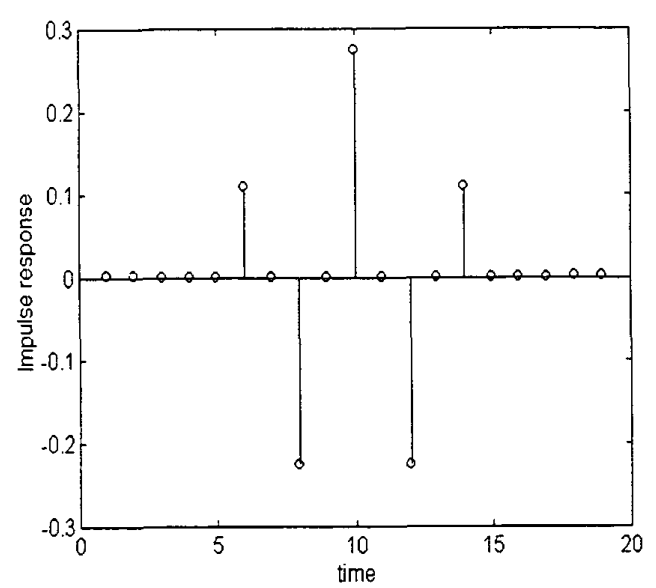

(b)

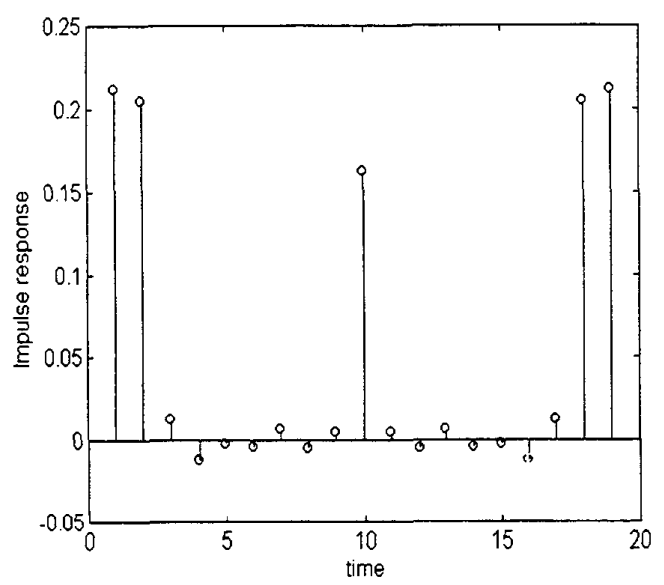

(c)

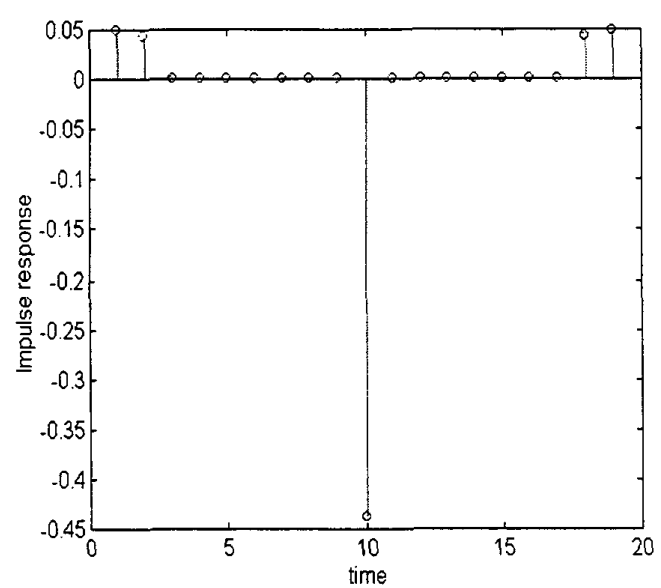

(d)

Fig.4 Time-domain responses of a five-user spread sprectrum CDMA coder

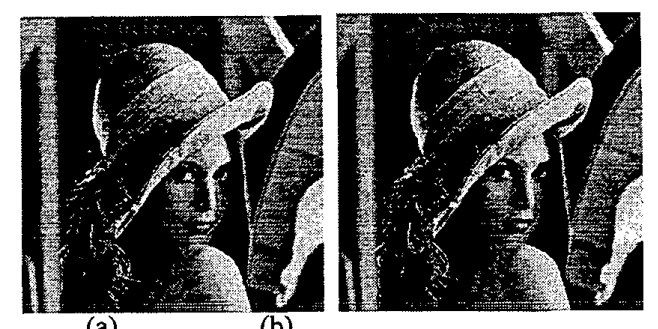

(a)

(b)

Fig. 5 a) original image b) watermarked image

\section{REFERENCES}

[1] P.P. Vaidyanathan, Multirate Systems and Filter Banks. Englewood Cliffs, NJ: Prentice-Hall, 1993.

[2] N. Akansu and M.J. Medley, Eds., Wavelet, Subband, and Block Transforms in Communication and Multimedia. Boston, MA: Kluwer 1998.

[3] N. Akansu and R. A. Haddad, Multiresolution Signal Decomposition Transforms, Subbands, and Wavelets. New York: Academic, 1992.

[4] L. Chen, K.P. Chan and T.Q. Nguyen, "A generalized algorithm for linear-phase paraunitary filter banks," IEEE Trans. on Signal Processing, vol. 46, no. 4, pp. 1154-1158, 1998.

[5] W.Kok, M.Ikehara, and T. Q. Nguyen, "Structures and factroizations of linear-phase paraunitary filter banks," in Proc. IEEE Int. Symp. Circuits Syst., June 1997, pp. I.365-I.368.

[6] K. Soman, P. P. Vaidyanathan, and T. Q. Nguyen, "Linear phase paraunitary filter banks," IEEE Trans. Signal Processing, vol. 41, pp. 3480-3496, Dec. 1993.

[7] I. J. Cox, J. Kilian, T. Leighton, and T. Shamoon, "Secure spread sprectrum watermarking for multimedia," IEEE Trans. Image Processing, vol. 6, no. 12, pp. 1673-1687, 1997. 